\title{
Specifying Urgency in Timed I/O Automata*
}

\author{
Biniam Gebremichael Frits Vaandrager \\ Nijmegen Institute for Computing and Information Sciences \\ Radboud University Nijmegen, The Netherlands \\ \{B.Gebremichael,F.Vaandrager\}@cs.ru.nl
}

\begin{abstract}
Sifakis et al advocate the use of deadline predicates for the specification of progress properties of Alur-Dill style timed automata. In this article, we extend these ideas to a more general setting, which may serve as a basis for deductive verification techniques. More specifically, we extend the TIOA framework of Lynch et al with urgency predicates. We identify a suitable language to describe the resulting timed $I / O$ automata with urgency and show that for this language time reactivity holds by construction. We also establish that the class of timed I/O automata with urgency is closed under composition. The use of urgency predicates is compared with three alternative approaches to specifying progress properties that have been advocated in the literature: invariants, stopping conditions and deadlines predicates. We argue that in practice the use of urgency predicates leads to shorter and more natural specifications than any of the other approaches. Some preliminary results on proving invariant properties of timed (I/O) automata with urgency are presented.
\end{abstract}

\section{Introduction}

In the literature on real-time systems there appears to be broad consensus on how to express quantitative timing constraints in state based modeling formalisms. Following the approach advocated by Alur and Dill [1], the idea is to designate certain state variables as clock variables. The values of these clock variables change as time advances. Also, clocks may be reset when discrete events occur. Timing constraints can be expressed, then, by conditions on clock values.

One issue on which there is no general consensus yet is how to specify progress properties, that is, properties which assert that a system must perform a certain action before a certain point in time. Merritt et al. in [17]

${ }^{*}$ This work was supported by the European Community Project IST-2001-35304 Advanced Methods for Timed Systems (AMETIST), http://ametist.cs.utwente.nl. 
propose a model with upper and lower bounds associated with tasks (that is, sets of system actions). In the work of Alur and Dill [1], progress is enforced via a Büchi style acceptance criterion: by requiring that some (sets of) locations are visited infinitely often the possibility is ruled out that a system stays in certain locations forever. A popular approach, which is advocated in $[9,2]$ and implemented in the tool UPPAAL [13], is to use (state) invariants. An invariant typically enforces a system action by limiting the amount by which time may advance in a given state. A related approach that is pursued in [11] is to use stopping conditions. Here the idea is that when a system reaches a state in which a stopping condition holds, time may not progress any further and a system action has to occur immediately. Sifakis and his colleagues $[20,4,19]$ advocate the use of deadlines for the specification of progress properties. Each transition of an Alur-Dill style timed automaton is decorated with an additional deadline predicate, which specifies when the transition becomes urgent. An advantage of deadline approach (which can be viewed as a generalization of the approach of [17]) is that under some reasonable assumptions, it ensures what is called time reactivity in [19] and timelock freedom in [5], that is, whenever time progress stops there exists at least one enabled transition. Under certain conditions, time reactivity is even preserved by parallel composition of automata $[5,19,3]$. The notion of deadlines has been incorporated in several modeling frameworks, see for instance $[5,7,21]$, and it has been implemented as part of the IF toolset [6].

The work of Sifakis et al $[20,4,19]$ takes place in a setting of AlurDill style timed automata, a system model that has limited expressivity in order to enable automatic state space exploration and model checking. In this article, we study the specification of progress properties in the much more general model of timed I/O automata (TIOA) of Lynch et al [11]. Even though fragments of the TIOA framework can be translated into timed automata [18], analysis of general TIOA models requires the use of deductive verification techniques and theorem provers such as PVS [10]. Inspired by the work of Sifakis et al, we introduce a similar notion of urgency predicates within the TIOA framework, both at the semantic level where we have infinite sets of states, transitions and trajectories, and at the syntactic level where system behavior is described finitely in terms of a logical language.

In the I/O automaton framework, transitions are typically specified using precondition/effect notation, that is, some type of guarded commands. This means that, for a given action name $b$ with parameters $\vec{h}$ a precondition predicate $\operatorname{pre}(\vec{v}, \vec{h})$ is given that defines from which states $\vec{v}$ action $b(\vec{h})$ is enabled, and an effect predicate $\operatorname{eff}\left(\vec{v}, \vec{h}, \vec{v}^{\prime}\right)$ that defines to which states $\vec{v}^{\prime}$ one may jump after doing action $b(\vec{h})$ in state $\vec{v}$. For the specification of timed systems we add a third predicate, the urgency predicate $\operatorname{urg}(\vec{v}, \vec{h})$, to every transition definition. The meaning of the urgency predicate is that if, 
for some $\vec{h}$, the state predicate

$$
\operatorname{pre}(\vec{v}, \vec{h}) \wedge \operatorname{urg}(\vec{v}, \vec{h})
$$

becomes true at a time point $t$ in a trajectory, then $t$ must be the limit time of that trajectory. Intuitively, the precondition specifies when a transition may occur, and the urgency predicate specifies when the transition becomes urgent, that is, either this or some other enabled discrete transition must occur immediately. A small but significant difference between our approach and the one of Sifakis et al $[20,19]$ is that Sifakis et al require that a deadline predicate implies the precondition predicate, whereas we achieve a similar effect by conjoining the urgency predicate with the precondition.

The main contributions of this article are:

1. Extension of the work of $[20,19,3]$ on deadline predicates to a much more expressive setting, which may serve as a basis for deductive verification techniques. More specifically, we extend the TIOA framework of [11] with urgency predicates at the semantic level, and define a suitable language to describe the resulting timed I/O automata with urgency. For this language, time reactivity holds by construction. We also establish that the class of TIOAs with urgency is closed under composition.

2. A comparison of urgency predicates with three alternative ways to specifying progress properties: invariants $[9,2]$, stopping conditions [11] and deadlines [20, 19,3]. Deadlines, stopping conditions and urgency predicates are shown to be (essentially) equally expressive. Invariants are slightly more expressive since they allow to bound the time at which an action occurs by a right open interval. Only use of urgency and deadline predicates gives time reactivity by construction. We argue that in practice the use of urgency predicates leads to shorter and more natural specifications than any of the other methods.

3. Some preliminary results on proving invariant properties of timed (I/O) automata with urgency.

\section{Timed (I/O) Automata with Urgency}

In this section, we describe our extension of the timed I/O automata framework of Lynch et al $[11,12]$ with urgency. In Subsections 2.1 and 2.2 we begin with recalling some definitions from $[11,12]$ : we introduce a basic vocabulary for describing timed behaviors and recall the notion of a timed automaton. In Subsection 2.3, we add a notion of urgent transitions to timed automata, both at the semantic and at the syntactic level. Next, in Subsection 2.4, an input/output distinction is added. Subsection 2.5, finally, 
defines a parallel composition operator and establishes that both the class of timed automata and the class of timed I/O automata with urgency are closed under composition. An example is presented that shows that timed automata with urgency are not closed under composition, in general.

\subsection{Describing Timed System Behavior}

In this section, we list the basic notions that are used in describing the behavior of a timed system, including both discrete and continuous changes. We simply sketch this material, leaving the reader to consult $[11,12]$ for the details.

The time domains we use is the set $\mathrm{R}$ of real numbers (in $[11,12]$ also other time domains are considered). States of automata will consist of valuations of variables. Each variable has both a static type, which defines the set of values it may assume, and a dynamic type, which gives the set of trajectories it may follow. We assume that dynamic types are closed under some simple operations: shifting the time domain, taking subintervals and pasting together intervals. We call a variable discrete if its dynamic type equals the pasting-closure of a set of constant-valued functions (i.e., the step-functions), and analog if its dynamic type equals the pasting-closure of a set of continuous functions (i.e., the piecewise-continuous functions).

A valuation for a set $V$ of variables is a function that associates with each variable $v \in V$ a value in its static type. We write $\operatorname{val}(V)$ for the set of all valuations for $V$. A trajectory for a set $V$ of variables describes the evolution of the variables in $V$ over time; formally, it is a function from a time interval that starts with 0 to valuations of $V$, that is, a trajectory defines a value for each variable at each time in the interval. We write $\operatorname{dom}(\tau)$ for the domain of trajectory $\tau$. A point trajectory is one with the trivial domain $\{0\}$. We write $\wp(\mathbf{x})$ for the point trajectory for valuation $\mathbf{x}$. The limit time of a trajectory $\tau, \tau$.ltime, is the supremum of the times in its domain. $\tau$.fval is defined to be the first valuation of $\tau$, and if $\tau$ is right-closed, $\tau$.lval is the last valuation. Suppose $\tau$ and $\tau^{\prime}$ are trajectories for $V$, with $\tau$ closed. The concatenation of $\tau$ and $\tau^{\prime}$, denoted by $\tau \frown \tau^{\prime}$, is the trajectory obtained by taking the union of the first trajectory and the function obtained by shifting the domain of the second trajectory until the start time agrees with the limit time of the first trajectory; the last valuation of the first trajectory, which may not be the same as the first valuation of the second trajectory, is the one that appears in the concatenation. Trajectory $\tau$ is a prefix of trajectory $\tau^{\prime}$, denoted $\tau \leq \tau^{\prime}$, if $\tau$ can be obtained by restricting $\tau^{\prime}$ to a subset of its domain. For every $t \in \operatorname{dom}(\tau)$, we define $\tau \unrhd t$ to be the trajectory obtained by taking the part of $\tau$ from $t$ onwards, and then shifting the domain so that it starts with 0 again. Formally, $\operatorname{dom}(\tau \unrhd t)=\{u \in \mathrm{R} \mid u+t \in \operatorname{dom}(\tau)\}$ and for all $u$ in the domain, $\tau \unrhd t(u)=\tau(u+t)$. 


\subsection{Timed Automata}

A timed automaton in the sense of $[11,12]$ is a state machine whose states are divided into variables and that has a set of discrete actions. The state of a timed automaton may change in two ways: by discrete transitions, which change the state atomically, and by trajectories, which describe the evolution of the state over intervals of time. Discrete transitions are labeled with actions, which are classified as either external or internal. The external actions are used to synchronize with the automaton's environment, while the internal actions are only visible to the automaton itself.

Formally, a timed automaton is a tuple $\mathcal{A}=(X, Q, \Theta, E, H, \mathcal{D}, \mathcal{T})$ with

- A set $X$ of internal variables.

- A set $Q \subseteq \operatorname{val}(X)$ of states.

- A nonempty set $\Theta \subseteq Q$ of start state.

- A set $E$ of external actions and a set $H$ of internal actions, disjoint from each other. We write $A \triangleq E \cup H$.

- A set $\mathcal{D} \subseteq Q \times A \times Q$ of discrete transition. An edge $e=\left(\mathbf{x}, a, \mathbf{x}^{\prime}\right) \in \mathcal{D}$, also written as $\mathbf{x} \stackrel{a}{\rightarrow} \mathbf{x}^{\prime}$, represent a transition from state $\mathbf{x}$ to $\mathbf{x}^{\prime}$ labeled with action $a$. We say that $a$ is enabled in $\mathbf{x}$ if $\mathbf{x} \stackrel{a}{\rightarrow} \mathbf{x}^{\prime}$ for some $\mathbf{x}^{\prime}$.

- A set $\mathcal{T}$ of trajectories for $X$ such that $\tau(t) \in Q$ for each $\tau \in \mathcal{T}$ and $t \in \operatorname{dom}(\tau)$. We require that the following axioms hold:

T0 (Existence of point trajectories)

If $\mathbf{x} \in Q$ then $\wp(\mathbf{x}) \in \mathcal{T}$.

$\mathbf{T} 1$ (Prefix closure)

For every $\tau \in \mathcal{T}$ and every $\tau^{\prime} \leq \tau, \tau^{\prime} \in \mathcal{T}$.

T2 (Suffix closure)

For every $\tau \in \mathcal{T}$ and every $t \in \operatorname{dom}(\tau), \tau \unrhd t \in \mathcal{T}$.

T3 (Concatenation closure)

Let $\tau_{0} \tau_{1} \tau_{2} \ldots$ be a sequence of trajectories in $\mathcal{T}$ such that, for each non final index, $i, \tau_{i}$ is closed and $\tau_{i} . l v a l=\tau_{i+1}$. fval. Then $\tau_{0} \frown \tau_{1} \frown \tau_{2} \ldots \in \mathcal{T}$.

A trajectory $\tau$ is maximal in $\mathcal{T}$ if there exists no $\tau^{\prime} \in \mathcal{T}$ with $\tau<\tau^{\prime}$. The following lemma (which as far as we know is new) states that each trajectory can be extended into a maximal one. Intuitively this is an obvious property, but the proof requires some work due to the fact that we know so little about $\mathcal{T}$.

Lemma 1 Let $\mathcal{A}$ be a timed automaton and let $\mathcal{T}$ be its set of trajectories. Then each trajectory in $\mathcal{T}$ is a prefix of a trajectory that is maximal in $\mathcal{T}$. 
Proof: Let $\tau \in \mathcal{T}$ be a trajectory. Suppose that $\tau$ can not be extended into a maximal trajectory. We derive a contradiction. Let $\tau_{0}=\tau, t_{0}=\tau_{0}$.ltime and let $u_{0}$ be the supremum of the limit times of the trajectories in $\mathcal{T}$ that extend $\tau$. Suppose $t_{0}=u_{0}$. Since $\tau_{0}$ is not maximal, this implies that it is right-open, and can be extended with a single state. But then the extended trajectory is maximal again, which is a contradiction. Hence $t_{0}<u_{0}$. Let $\tau_{1}$ be a trajectory that extends $\tau_{0}$ such that if $u_{0}=\infty$ then $t_{1}=\tau_{1}$. ltime $=$ $t_{0}+1$ else $t_{1}=\tau_{1}$.ltime $>\frac{t_{0}+u_{0}}{2}$. Let $u_{1}$ be the supremum of the limit times of the trajectories in $\mathcal{T}$ that extend $\tau_{1}$. Then $u_{1} \leq u_{0}$. Continuing the construction, we find an infinite chain of trajectories $\tau_{0}<\tau_{1}<\tau_{2}<\cdots$ and real numbers $t_{i}, u_{i}$ such that

$$
t_{0}<t_{1}<t_{2}<\cdots u_{3} \leq u_{2} \leq u_{1} \leq u_{0} .
$$

In addition, we know that (1) there exists a $k$ such that for all $j>k$, $u_{j}<\infty$ (otherwise it would be possible to extend $\tau_{0}$ into an infinite and hence maximal trajectory), (2) $t^{\prime}=\lim _{i \rightarrow \infty} t_{i}=\lim _{i \rightarrow \infty} u_{i}$. Let $\tau^{\prime}$ be the limit of the trajectories $\tau_{i}$. Then $t^{\prime}=\tau^{\prime}$.ltime. We know that $\tau^{\prime} \in \mathcal{T}$ by axiom T3 (concatenation closure). By assumption, since $\tau<\tau^{\prime}, \tau^{\prime}$ is not maximal in $\mathcal{T}$. Hence there exists a trajectory $\tau^{\prime \prime}$ with $\tau^{\prime}<\tau^{\prime \prime}$ and $t^{\prime}<\tau^{\prime \prime}$.ltime $=t^{\prime \prime}$. But this contradicts the fact that, for sufficiently large $k$, the supremum of the limit times of the trajectories that extend $\tau_{k}$ is strictly less than $t^{\prime \prime}$.

\subsection{Adding Urgency}

We now extend timed automata extra state predicates, urgency predicates, one for each action.

A timed automaton with urgency is a pair $(\mathcal{A}, U)$ of a timed automaton $\mathcal{A}=(X, Q, \Theta, E, H, \mathcal{D}, \mathcal{T})$ and an urgency predicate $U: Q \times A \rightarrow$ Bool. If $U(\mathbf{x}, a)=$ true then we say that action $a$ is potentially urgent in a state $\mathbf{x}$. Action $a$ is urgent in state $\mathbf{x}$ if it is potentially urgent and in addition enabled. We require that the following two axioms hold:

T4 (Urgency)

For every $\tau \in \mathcal{T}, t \in \operatorname{dom}(\tau)$ and $a \in A$ : if $a$ is urgent in $\tau(t)$ then $t=\tau$.ltime.

T5 (Maximality)

For every $\tau \in \mathcal{T}$, if $\tau$ is maximal and finite then $\tau$ is right-closed and some $a \in A$ is urgent in $\tau$.lval.

Axiom T4 states that as soon as an action becomes urgent, this action or some other action that is enabled has to occur immediately. Axiom T5 states that each maximal and finite trajectory enables an urgent action at the end. 
Timed automata with urgency can be conveniently specified in a slight variation of the TIOA language [10].

Example 1 To illustrate this language, we consider the simple model of a train displayed in Figure 1. The automaton runs cyclically through states start, light and gate. After spending between $(2,5]$ time units in start the automaton jumps to light, then within $(5,10]$ time units after arrival in light the automaton jumps to gate, and after exactly 2 time units in gate the automaton returns to the initial state start.

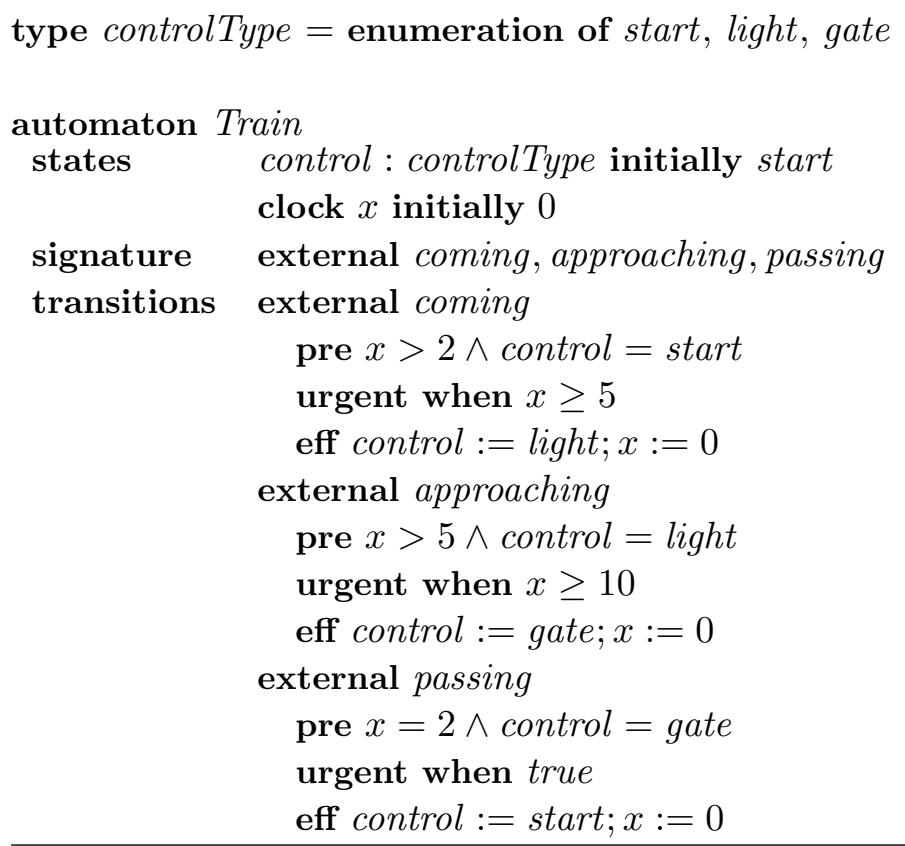

Figure 1: A simple model of a train.

The definitions of the signature, state variables, initial states, and transition in our language are similar to their counterparts in the IOA language. We refer to the IOA user guide and reference manual [8] for additional information on this part of the language. ${ }^{1}$ In this article, we consider only two types of state variables, which differ in their dynamic types: discrete variables (such as control), whose value remains unchanged along a trajectory, and clocks (such as $x$ ), which are real-valued variables whose value increases with rate 1 along a trajectory. ${ }^{2}$ The set of states consists of all valuations of the state variables $\vec{v}$. At the syntactic level, we have a finite

\footnotetext{
${ }^{1}$ Since the emphasis in this article is on urgency we decided not to present all datatype definitions. At this point, our specifications are (deliberately) a bit sloppy.

${ }^{2}$ Our results easily generalize to more general dynamic types and continuous behavior defined by arbitrary differential equations and inclusions, such as studied e.g. in [11, 14].
} 
number of action names $b$ and each action name comes with a list $\vec{h}$ of formal parameters. At the semantic level, the set of actions consists of pairs of an action name $b$ and a valuation $\mathbf{h}$ of the parameters $\vec{h}$. The transition relation is defined via a finite number of transition definitions. Each transition definition consists of an action name $b$, a list $\vec{h}$ of formal parameters, a precondition predicate $\operatorname{pre}(\vec{v}, \vec{h})$ that defines from which states an action $b(\vec{h})$ is enabled, an urgent when predicate $\operatorname{urg}(\vec{v}, \vec{h})$ that specifies when that action becomes urgent, and an effect predicate eff $\left(\vec{v}, \vec{h}, \vec{v}^{\prime}\right)$ specifying to which states $\vec{v}^{\prime}$ one may jump after doing action $b(\vec{h})$ in state $\vec{v}$. If no parameters are mentioned then the parameter list is assumed to be empty, if no precondition is mentioned then it is implicitly assumed to equal true, and if no urgency predicate is mentioned this is assumed to equal false. We further assume that the effect relation is total, in the sense that for each state $\mathbf{x}$ and parameter valuation $\mathbf{h}$ such that $\operatorname{pre}(\mathbf{x}, \mathbf{h})$ holds, there exists at least one state $\mathbf{x}^{\prime}$ such that eff $\left(\mathbf{x}, \mathbf{h}, \mathbf{x}^{\prime}\right)$ holds. If the effect predicate is defined using (deterministic) assignments, such as in Figure 1, this property trivially holds.

The set of trajectories is defined implicitly. For $\mathbf{x}$ a state and $t$ a nonnegative real number, let $\mathbf{x} \oplus t$ be the state given by

$$
\mathbf{x} \oplus t(v) \triangleq \begin{cases}\mathbf{x}(v) & \text { if } v \text { is discrete } \\ \mathbf{x}(v)+t & \text { if } v \text { is a clock. }\end{cases}
$$

The state $\mathbf{x} \ominus t$ is defined similarly: replace + by - in the definition of $\oplus$. For $\mathbf{x}$ a state and $I$ a time interval that starts with 0 , a pretrajectory from $\mathbf{x}$ over $I$ is a function $\tau: I \rightarrow Q$ such that for each $t \in I, \tau(t)=\mathbf{x} \oplus t$. The set of trajectories is defined to be the set of all pretrajectories $\tau$ satisfying that if some action $a$ is urgent in some state $\tau(t)$, for $t$ in the domain of $\tau$, $t=\tau$.ltime.

Example 2 Figure 2 gives another example of a specification in our language. It is a model of a reliable FIFO channel that delivers its messages within a certain time bound, represented by the automaton parameter $b$, which is a positive real number. The other automaton parameter $M$ represents the type of messages communicated by the channel. The states of the automaton are valuations of the state variables queue and now. The discrete variable queue holds a finite sequence of pairs consisting of a message that has been sent and its delivery deadline. The clock variable now records the current real time. A send $(m)$ action, which is always enabled and never becomes urgent, adds to the queue a new pair whose first component is $m$ and whose second component is the deadline now $+b$. A receive $(m)$ action can occur when $m$ is the first message in the queue and it results in the removal of the first message from the queue. The receive $(m)$ action becomes urgent when the delivery deadline $u$ of the first message equals the current time now. 


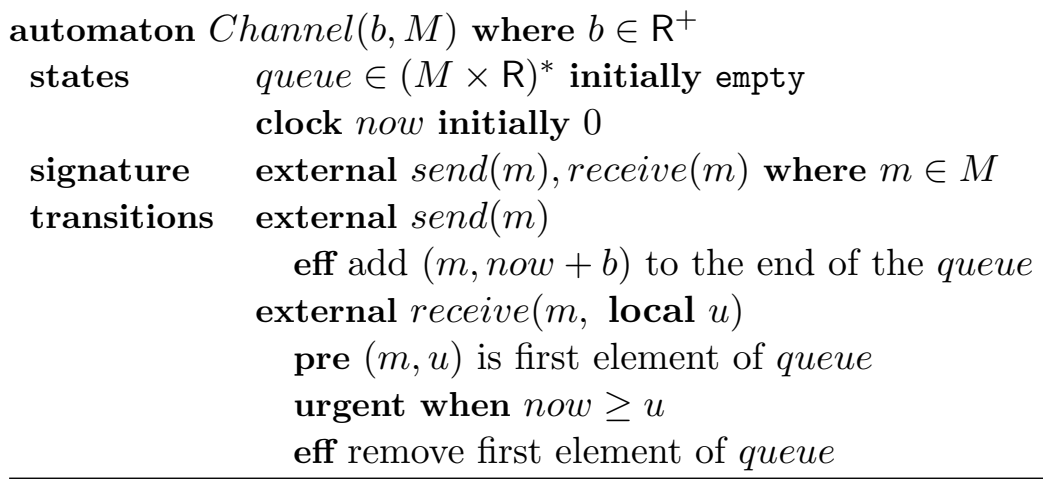

Figure 2: Time-bounded channel.

By construction the set of trajectories denoted by a specification in our language satisfies axioms T0-T4. However, the example below shows that axiom $\mathbf{T 5}$ does not need to hold in general.

Example 3 The timed automaton specified in Figure 3 has a transition with precondition $x>4 \wedge b=$ false and urgency predicate true. Axiom T5 does not hold, since time can only advance up to $x=4$ but at that time the transition is not (yet) enabled.

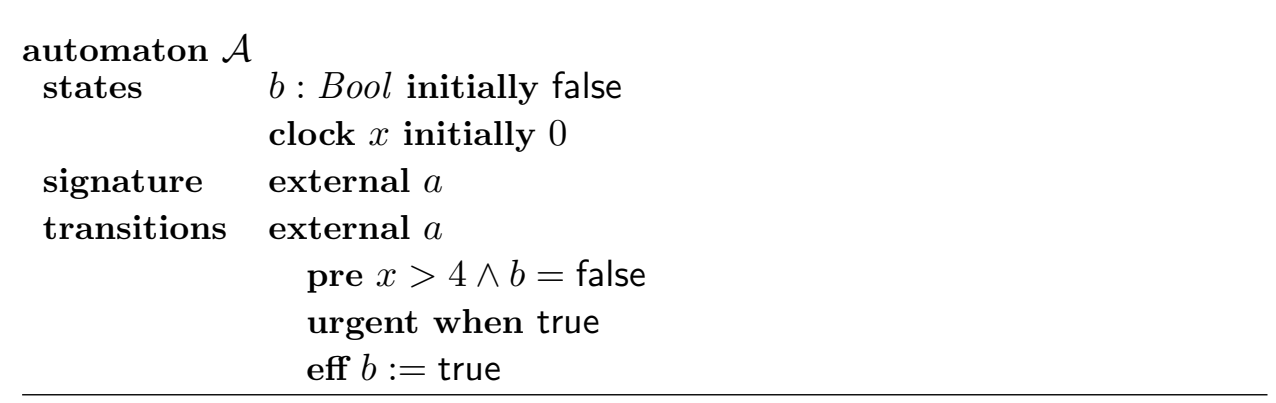

Figure 3: A counterexample to axiom T5.

In order to avoid the counterexample of Figure 3, it is sufficient that certain predicates derived from the transition definitions are left-closed in the sense of [3]. For each transition definition $t r$

$$
\begin{aligned}
& b(\vec{h}) \\
& \text { pre } \operatorname{pre}(\vec{v}, \vec{h}) \\
& \text { urgent when } \operatorname{urg}(\vec{v}, \vec{h}) \\
& \text { eff } \operatorname{eff}\left(\vec{v}, \vec{h}, \vec{v}^{\prime}\right)
\end{aligned}
$$

let predicate $U r g(t r)$ be given by

$$
\operatorname{Urg}(\operatorname{tr})(\vec{v}, \vec{h}) \triangleq \exists \vec{h}: \operatorname{pre}(\vec{v}, \vec{h}) \wedge \operatorname{urg}(\vec{v}, \vec{h})
$$


Following [3], we define a state predicate $\varphi$ to be left-closed if, for all $\vec{v}$,

$$
\neg \varphi(\vec{v}) \Longrightarrow \exists \epsilon>0 \forall \epsilon^{\prime} \leq \epsilon: \neg \varphi\left(\vec{v} \oplus \epsilon^{\prime}\right)
$$

In practice, left-closedness can be easily obtained by only using non-strict lower bounds on clocks. For instance, $x \geq 4 \wedge b=$ false is left-closed but $x>4 \wedge b=$ false is not. We can now formally state the following theorem.

Theorem 1 If the predicate $\bigvee_{t r}$ Urg $(t r)$ is left-closed then axiom $\mathbf{T 5}$ holds.

Proof: Suppose that $\tau$ is a maximal and finite trajectory.

Assume that the domain of $\tau$ is right-open. Then, by T4, nowhere on $\tau$ an action becomes urgent. But this means that the extension of $\tau$ with a single state at the end gives a legal trajectory, thus contradicting the assumption that $\tau$ is maximal. Hence, without loss of generality, we may assume that the domain of $\tau$ is right-closed.

Assume that no action $a$ is urgent in $\tau$.lval. This means that the disjunction $\bigvee_{t r} \operatorname{Urg}(t r)$ does not hold in $\mathbf{x}$. But this means that there exists a small $\epsilon$-extension of $\tau$ in which no action $a$ is urgent. This extension is then a legal trajectory, which contradicts again our assumption that $\tau$ is maximal.

\subsection{Adding an I/O Distinction}

In this section, we further refine the model of timed automata by distinguishing between input and output actions as in [11].

A timed I/O automaton with urgency is a quadruple $(\mathcal{A}, U, I, O)$ where

- $(\mathcal{A}, U)$ is a timed automaton with urgency, with $\mathcal{A}=(X, Q, \Theta, E, H, \mathcal{D}, \mathcal{T})$.

- $I$ and $O$ partition $E$ into input and output actions, that is $E=I \cup O$ and $I \cap O=\emptyset$. Actions in $H \cup O$ are called locally controlled. We write $L \triangleq H \cup O$ and $A \triangleq E \cup H$.

We require that the following axiom holds:

E0 (Inputs not urgent)

For every $\mathbf{x} \in Q$ and every $a \in I, U(\mathbf{x}, a)=$ false.

E1 (Input action enabling)

For every $\mathbf{x} \in Q$ and every $a \in I$, there exists $\mathbf{x}^{\prime} \in Q$ such that $\left(\mathbf{x}, a, \mathbf{x}^{\prime}\right) \in \mathcal{D}$.

The input actions are assumed not to be under the automaton's controlthey just arrive from the outside - while the automaton itself specifies what output and internal actions should be performed. In line with these intuitions, axiom E0 states that input actions never become urgent. Axiom E1 
is the usual input enabling condition of ordinary I/O automata [15]; it says that a TIOA with urgency is able to accomodate an input action whenever it arrives. At the syntactic level, a sufficient condition for axioms E0 and E1 to hold is that, in each transition definition for an input action, the precondition is true and the urgency predicate is false.

A desirable property for models of real-time systems is time reactivity. This means that in each state, either time is allowed to advance forever, or time may advance for a while up to a point where the system is prepared to react with some locally controlled action. In [11], an axiom E2 is required for timed I/O automata which captures this property:

E2 (Time-passage enabling)

For every $\mathbf{x} \in Q$, there exists $\tau \in \mathcal{T}$ such that $\tau$.fval $=\mathbf{x}$ and either

- $\tau$.ltime $=\infty$, or

- $\tau$ is right-closed and some locally controlled action $l \in L$ is enabled in $\tau . l v a l$.

For a TIOA with urgency, time reactivity is implied by the other axioms.

Theorem 2 Each timed I/O automaton with urgency satisfies axiom E2.

Proof: Assume that $\mathbf{x}$ is a state. Then, by axiom $\mathbf{T} \mathbf{1}, \wp(\mathbf{x})$ is a trajectory in $\mathcal{T}$. By Lemma 1 there exists a maximal trajectory $\tau$ that extends $\wp(\mathbf{x})$. By construction, $\tau$.fval $=\mathbf{x}$. By axiom T5 either

- t.ltime $=\infty$ which completes the proof, or

- $\tau$ is right-closed and there is an action $a$ that is urgent in $\mathbf{x}^{\prime}=\tau$.lval. By axiom E0 this cannot be an input action. Hence $\mathbf{x}^{\prime}$ enables a locally controlled action, as required.

\subsection{Composition}

We say that timed automata $\mathcal{A}_{1}$ and $\mathcal{A}_{2}$ are compatible if they have no state variables in common, and if neither automaton has an internal action that is an action of the other automaton. If $\mathcal{A}_{1}$ and $\mathcal{A}_{2}$ are compatible then their composition $\mathcal{A}_{1} \| \mathcal{A}_{2}$ is defined formally to be the timed automaton. $\mathcal{A}=(X, Q, \Theta, E, H, \mathcal{D}, \mathcal{T})$ where

- $X=X_{1} \cup X_{2}$.

- $Q=\left\{\mathbf{x} \in \operatorname{val}(X) \mid \mathbf{x}\left\lceil X_{i} \in Q_{i}, i \in\{1,2\}\right\}\right.$. 
- $\Theta=\left\{\mathbf{x} \in Q \mid \mathbf{x}\left\lceil X_{i} \in \Theta_{i}, i \in\{1,2\}\right\}\right.$.

- $E=E_{1} \cup E_{2}$ and $H=H_{1} \cup H_{2}$.

- For each $\mathbf{x}, \mathbf{x}^{\prime} \in Q$ and each $a \in A, \mathbf{x} \stackrel{a}{\rightarrow} \mathcal{A} \mathbf{x}^{\prime}$ iff for $i \in\{1,2\}$, either (1) $a \in A_{i}$ and $\mathbf{x}\left\lceil X_{i} \stackrel{a}{\rightarrow}_{i} \mathbf{x}^{\prime}\left\lceil X_{i}\right.\right.$, or (2) $a \notin A_{i}$ and $\mathbf{x}\left\lceil X_{i}=\mathbf{x}^{\prime}\left\lceil X_{i}\right.\right.$.

- $\tau \in \mathcal{T} \Leftrightarrow \tau \downarrow X_{i} \in \mathcal{T}_{i}, i \in\{1,2\}$.

We refer to [12] for a proof that $\mathcal{A}_{1} \| \mathcal{A}_{2}$ is a timed automaton indeed, that is, the above structure satisfies axioms T0-T3.

Two timed automata with urgency, $\left(\mathcal{A}_{1}, U_{1}\right)$ and $\left(\mathcal{A}_{2}, U_{2}\right)$, are compatible if the underlying timed automata $\mathcal{A}_{1}$ and $\mathcal{A}_{2}$ are compatible. In this case, the composition is defined to be the structure $(\mathcal{A}, U)$, where $\mathcal{A}=\mathcal{A}_{1} \| \mathcal{A}_{2}$ and $U$ is given by

$$
U\left(\left(\mathbf{x}_{1}, \mathbf{x}_{2}\right), a\right)=U_{1}\left(\mathbf{x}_{1}, a\right) \vee U_{2}\left(\mathbf{x}_{2}, a\right),
$$

where by convention $U_{i}\left(\mathbf{x}_{i}, a\right)=$ false if $a$ is not in the signature of $\mathcal{A}_{i}$. So an action is urgent in a state of the composed system iff it is urgent in one of the component states. In general, the composition is not a timed automaton with urgency. The problem is due to axiom T5: if, for instance, we compose a system in which action $a$ becomes urgent at time 1 with a system that has $a$ in its signature but without any $a$-transition, then the composed system has a maximal trajectory of length 1 in which no transition is enabled. Several papers address the issue of how timelock freedom (or more generally, liveness) can be preserved by composition, see for instance $[5,19,3]$. In this article, we present one simple but useful result along these lines: the class of timed I/O automata with urgency is closed under composition.

We say that two timed I/O automata with urgency, $\left(\mathcal{A}_{1}, U_{1}, I_{1}, O_{1}\right)$ and $\left(\mathcal{A}_{2}, U_{2}, I_{2}, O_{2}\right)$, are compatible if the underlying timed automata $\mathcal{A}_{1}$ and $\mathcal{A}_{2}$ are compatible, and also they have no output actions in common. A consequence of these conditions is that each action is controlled by at most one component. In this case, the composition is defined to be the structure $(\mathcal{A}, U, I, O)$, where $(\mathcal{A}, U)$ is the composition of $\left(\mathcal{A}_{1}, U_{1}\right)$ and $\left(\mathcal{A}_{2}, U_{2}\right), I=$ $\left(I_{1} \cup I_{2}\right)-\left(O_{1} \cup O_{2}\right)$, and $O=O_{1} \cup O_{2}$. That is, an external action of the composition is classified as an output if it is an output of one of the component automata, otherwise it is classified as an input.

Theorem 3 The composition of two compatible timed I/O automata with urgency is again a timed I/O automaton with urgency.

Proof: Straightforward from the definitions. Axiom T5 holds because if $\tau$ is a maximal and finite trajectory of the composition there exists at least one component such that the projection of $\tau$ on that component is maximal. Using T5 for the component gives that the projection is right-closed and 
that some action $a$ of the component is urgent in the final state. By axiom E0 we know that $a$ is a locally controlled action. We infer that $\tau$ is rightclosed and (by axiom E1 for the other component) that $a$ is urgent in the composed system in the final state of $\tau$.

\section{Expressivity}

In this section, we compare the expressivity of urgency predicates with that of the deadline predicates of $[20,19]$, the stopping conditions of [11], and the invariants as used e.g. in $[9,2,13]$.

\subsection{Deadline Predicates}

Instead of using urgency predicates, we could follow the approach of Sifakis et al $[20,19]$ even more closely by using deadline predicates. This would mean that, for a given action name $b$ with parameters $\vec{h}$, besides the precondition $\operatorname{pre}(\vec{v}, \vec{h})$ and the effect $\operatorname{eff}\left(\vec{v}, \vec{v}^{\prime}, \vec{h}\right)$, also a deadline predicate $d l(\vec{v}, \vec{h})$ is specified such that $d l(\vec{v}, \vec{h}) \Longrightarrow \operatorname{pre}(\vec{v}, \vec{h})$ holds. The semantics of a deadline predicate is that if, for some $\vec{h}$, the state predicate

$$
d l(\vec{v}, \vec{h})
$$

becomes true at a time point $t$ in a trajectory, then $t$ must be the limit time of that trajectory.

Clearly, any definition of a timed automaton with urgency predicates can be transformed into an equivalent (in the sense that the defined automata are semantically equal) definition with deadline predicates by replacing each urgency predicate $\operatorname{urg}(\vec{v}, \vec{h})$ by a deadline predicate $\operatorname{pre}(\vec{v}, \vec{h}) \wedge \operatorname{urg}(\vec{v}, \vec{h})$. Conversely, any definition with deadline predicates can be transformed into an equivalent definition with urgency predicates by replacing each deadline predicate $d l(\vec{v}, \vec{h})$ by an identical urgency predicate $d l(\vec{v}, \vec{h})$.

Study of the examples in Figure 1 and Figure 2, and the examples in [12] indicates that the use of urgency predicates leads to slightly shorter specifications than the use of deadlines.

\subsection{Stopping Conditions}

Another alternative for urgency predicates are the stopping conditions as used in [11]. A stopping condition is a state predicate $s c(\vec{v})$ such that if $s c(\vec{v})$ becomes true at a time point $t$ in a trajectory, then $t$ must be the limit time of that trajectory.

We again checked the examples from Figure 1, Figure 2 and [12], and in each case urgency predicates lead to shorter and (in our view) more natural specifications than stopping conditions. Figure 4, for instance, shows how 
the transitions and trajectories of the example of Figure 1 can be rewritten using a stopping condition. The disadvantages should be clear: upper bounds are no longer specified next to the corresponding lower bounds, and parts of the preconditions have to be repeated in the stopping condition.

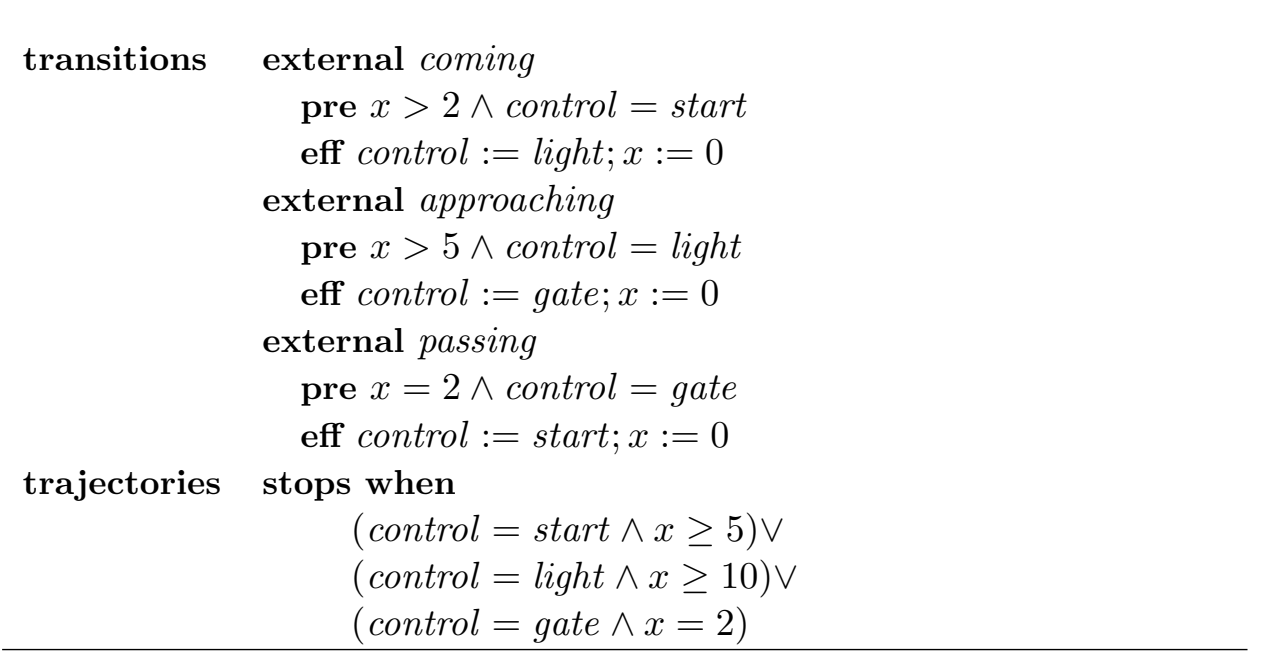

Figure 4: The train model defined using a stopping condition.

Any definition of a timed automaton with urgency predicates can be transformed into an equivalent definition with stopping conditions by replacing the urgency predicates by a single stopping condition that is the disjunction of the formula pre $(\vec{v}, \vec{h}) \wedge \operatorname{urg}(\vec{v}, \vec{h})$, for all transition definitions.

Stopping conditions are more expressive than urgency predicates since they allow one to define timed automata that are not time reactive and in which "the universe" may come to a halt. Figure 5 gives an example. Of

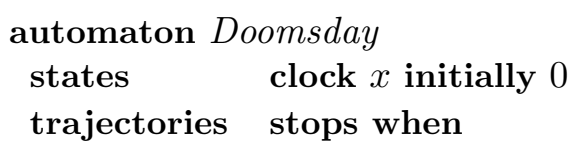

Figure 5: A time deadlock.

course this is a form of additional expressivity that we would rather not have! For a timed automaton definition with a stopping condition $s c(\vec{v})$ it seems reasonable to require that the following variation of axiom T5 holds:

T5' (Maximality)

For every $\tau \in \mathcal{T}$, if $\tau$ is maximal and finite then $\tau$ is right-closed, $s c(\tau . l v a l)$ and some (locally controlled) $a \in A$ is enabled in $\tau . l v a l$.

If this property holds, the specification can be transformed into an equivalent specification with urgency predicates: in case there is no I/O distinction we 
just add an urgency predicate $s c(\vec{v})$ to each transition definition, if there is an I/O distinction we add urgency predicate $s c(\vec{v})$ to each locally controlled transition and urgency predicate false to each input transition.

\subsection{Invariants}

A popular way to specify progress properties, which has been advocated in $[9,2]$ and implemented in UPPAAL [13], is the use of invariants. An invariant is a state predicate $i n v(\vec{v})$ that is required to hold for all states along all trajectories. Figure 6 shows how the transitions and trajectories of the example of Figure 1 look with invariants. Like stopping conditions also

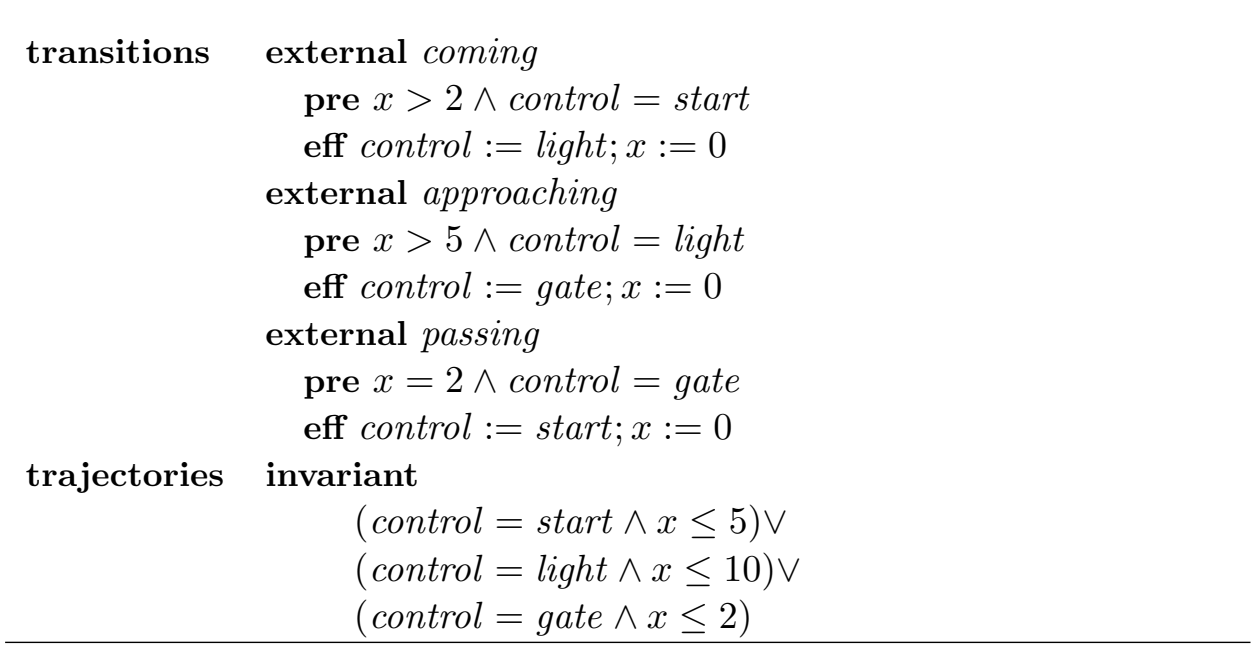

Figure 6: The train model defined with an invariant.

invariants allow one to define timed automata that are not time reactive, a clear disadvantage of these specification styles. The example of Figure 5, for instance, can easily be encoded using invariants (replace the stopping condition by an invariant $x \leq 1$ ).

Invariants also allow one to specify strict upper bounds on the timing of events, as illustrated in Figure 7 . The same timed automaton can not be specified using urgency predicates, for the simple reason that it has a maximal trajectory that is right-open, which is in violation with axiom T5. If we are willing to consider timed automata up to some suitable equivalence (for instance, the trace equivalence defined in [11]) then it is possible to specify strict upper bounds with urgency predicates, but this requires the use of auxiliary variables and unbounded nondeterminism. Figure 8 illustrates the specification of a strict upper bound with an urgency predicate. The idea is to choose nondeterministically a value in the interval $[0,1)$ and then make $a$ urgent when time has reached this value. Apart from the fact that the second specification is less intuitive, the use of unbounded nondeterminism 


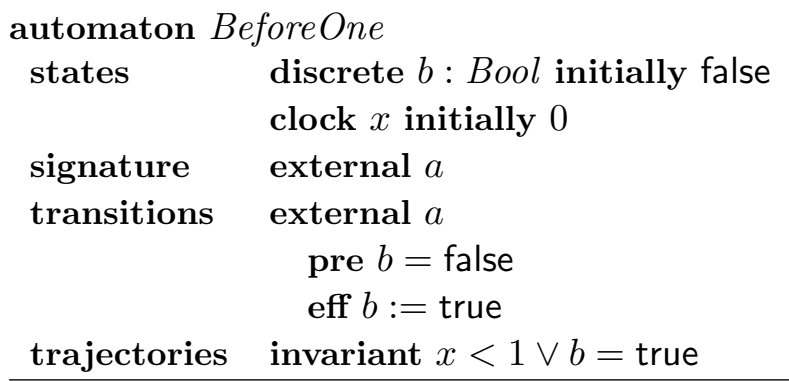

Figure 7: Specification of a strict upper bound on timing with an invariant.

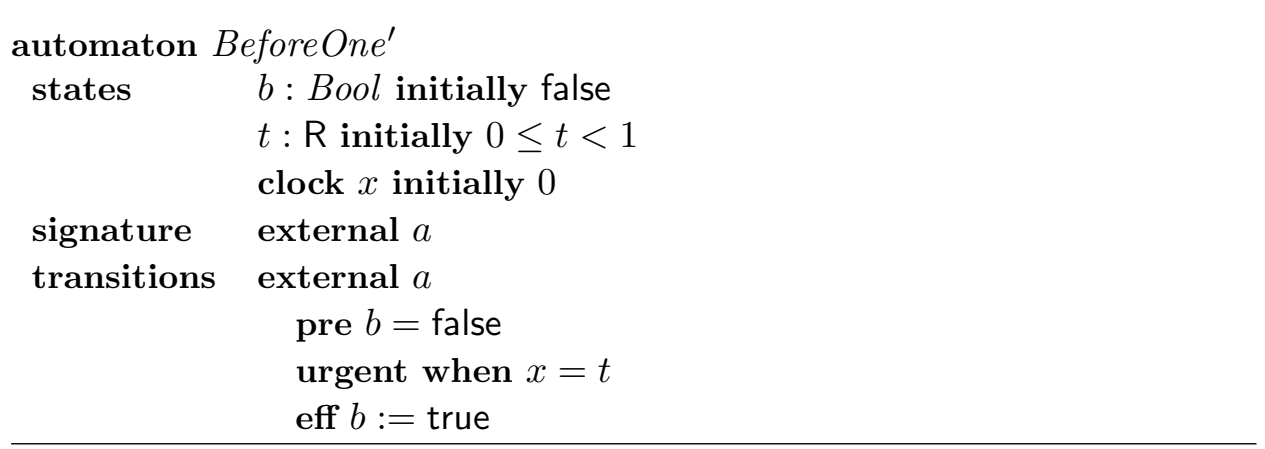

Figure 8: Specification of a strict upper bound on timing with urgency.

will constitute a serious obstacle for automatic verification methods. In all practical applications of timed automata that we are aware of, the use of only non strict upper bounds on timing is not a restriction. For applications where use of strict upper bounds is essential, use of invariants is probably more appropriate than use of urgency predicates.

Timed automata with urgency predicates can (in many cases) be translated to equivalent timed automata with invariants. Robson [18] describes how a fragment of TIOA with urgency predicates can be translated to the input language of UPPAAL. ${ }^{3}$ Below we discuss a more general translation scheme. We say that a state predicate $\varphi(\vec{v})$ is stable (under time progress) if

$$
\varphi(\vec{v}) \Longrightarrow \forall d>0: \varphi(\vec{v} \oplus d)
$$

Typically, a predicate will be stable if it only involves lower bounds on clocks and no upper bounds. The lower hull of state predicate $\varphi(\vec{v})$ is the set of

\footnotetext{
${ }^{3}$ The UPPAAL syntax for invariant predicates is rather restricted. For each individual location the invariant is a conjunction of conditions of the form $x \leq e$ or $x<e$ where $x$ is a clock and $e$ is an expression that evaluates to an integer. This restriction forces Robson to split locations as part of her translation.
} 
valuations given by

$$
L H(\varphi) \triangleq\left\{\mathbf{x} \mid \varphi(\mathbf{x}) \wedge \exists \epsilon>0 \forall 0<\epsilon^{\prime} \leq \epsilon: \neg \varphi\left(\mathbf{x} \ominus \epsilon^{\prime}\right)\right\}
$$

The upper hull of a state predicate can be defined similarly, just replace $\ominus$ by $\oplus$ in the above definition. If $\varphi$ only involves (non-strict) lower bounds on clocks then the lower hull can easily be expressed again as a predicate by replacing the $\geq$ signs with $=$.

Now consider a definition of a timed automaton with urgency predicates such that all predicates $\operatorname{Urg}(t r)$ are left-closed and stable. An equivalent timed automaton with invariants can be obtained by replacing the urgency predicates with the invariant

$$
i n v=\neg\left(\bigvee_{t r} \operatorname{Urg}(t r)\right) \vee L H\left(\bigvee_{t r} \operatorname{Urg}(t r)\right),
$$

provided that the state predicate $i n v$ holds initially and after each discrete transition, i.e.,

$$
\operatorname{pre}(\vec{v}, \vec{h}) \wedge \operatorname{eff}\left(\vec{v}, \vec{h}, \vec{v}^{\prime}\right) \Longrightarrow \operatorname{inv}\left(\vec{v}^{\prime}\right) .
$$

The proof of the equivalence is straightforward and left to the reader.

Any timed automaton definition with right-closed invariants can be easily translated to a timed automaton with stopping conditions: the stopping condition is defined to be (a predicate denoting) the upper hull of the invariant. The translation scheme of Section 3.2 can then be used (provided axiom $\mathbf{T} 5$ ' holds) to translate the resulting timed automaton with stopping conditions to a timed automaton with urgency.

\section{Proving Invariant Properties}

In this section, we discuss how to establish invariant properties for specifications that involve urgency predicates. It is important to distinguish invariant properties from the invariant assertions that were discussed in the previous section as a construct to specify progress. An invariant property is a state predicate that holds for all reachable states of a given system. An invariant in the sense of previous section is an assertion that is actually used to define (the trajectories of) a system. Any invariant in the sense of the previous section is actually an invariant property of the system that it helps to define. The converse implication typically does not hold.

An execution fragment of a timed automaton $\mathcal{A}$ is a sequence $\alpha=$ $\tau_{0} a_{1} \tau_{1} a_{2} \tau_{2} \ldots$, where each $a_{i}$ is an action of $\mathcal{A}$, each $\tau_{i}$ is a trajectory of $\mathcal{A}$, and for every $i, \tau_{i}$.lval $\stackrel{a_{i+1}}{\rightarrow} \tau_{i+1}$.fval. An execution fragment records what happens during a particular run of a system, including all the discrete state changes and all the changes that occur while time advances. An $e x-$ ecution is an execution fragment whose first state is a start state of $\mathcal{A}$. A 
state is reachable in $\mathcal{A}$ if it is the last state of the last trajectory of a finite execution of $\mathcal{A}$. A state predicate $\varphi$ is an invariant of $\mathcal{A}$ if it holds for all reachable states of $\mathcal{A}$.

In order to prove that an assertion $\varphi$ is an invariant of $\mathcal{A}$, it suffices to prove that it holds initially and is preserved by all discrete transitions as well as by all time $(d)$ steps defined by

$$
\mathbf{x} \stackrel{\text { time }(d)}{\rightarrow} \mathbf{x}^{\prime} \triangleq \exists \tau \in \mathcal{T}: \tau \text {.fval }=\mathbf{x} \wedge \tau \text {.ltime }=d \wedge \tau . \text { lval }=\mathbf{x}^{\prime} .
$$

If we manage to give a simple and tractable characterization of the time $(d)$ predicate, then all the invariant proof techniques which are presented (for instance) in [16] become available in our setting.

Let $t r$ be a transition definition for an action name $b$ with parameter $\vec{h}$ with precondition pre $(\vec{v}, \vec{h})$, urgency predicate $\operatorname{urg}(\vec{v}, \vec{h})$, and effect predicate eff $\left(\vec{v}, \vec{h}, \vec{v}^{\prime}\right)$. For $d \geq 0$, the time progress predicate $t p(\vec{v}, t r, d)$ expresses that transition $t r$ permits time to advance with an amount $d$ from state $\vec{v}$. The predicate is formally defined in terms of the $\operatorname{Urg}(\operatorname{tr})$ predicate of (2):

$$
t p(\vec{v}, t r, d) \triangleq \forall 0 \leq e<d: \neg \operatorname{Urg}(\operatorname{tr})(\vec{v} \oplus e, \vec{h})
$$

which is equivalent to

$$
t p(\vec{v}, t r, d) \triangleq \forall 0 \leq e<d \forall h: \operatorname{pre}(\vec{v} \oplus e, \vec{h}) \Longrightarrow \neg u r g(\vec{v} \oplus e, \vec{h})
$$

Using the time progress predicates, we characterize the time advance steps time $(d)$ as follows:

$$
\begin{aligned}
& \text { time }(d) \\
& \quad \text { pre } \bigwedge_{t r} t p(\vec{v}, t r, d) \\
& \quad \text { eff } \vec{v}:=\vec{v} \oplus d
\end{aligned}
$$

In many cases it is possible to simplify the time progress predicates, by eliminating the universal quantifications from their definition. As an example, consider the timed automaton of Figure 1. The time progress predicate for the coming transition is

$$
\forall 0 \leq e<d: \neg(x+e>2 \wedge \text { control }=\text { start } \wedge x+e \geq 5)
$$

which is equivalent to

$$
\forall 0 \leq e<d: \neg(\text { control }=\text { start } \wedge x+e \geq 5)
$$

which is equivalent to

$$
\neg(\text { control }=\text { start } \wedge x+d>5)
$$

which is equivalent to

$$
\text { control }=\text { start } \Longrightarrow x+d \leq 5
$$


Similarly, the time progress predicates for the approaching and passing transitions can be written resp. as

$$
\begin{gathered}
\text { control }=\text { light } \Longrightarrow x+d \leq 10 \\
\text { control }=\text { gate } \Longrightarrow x+d \leq 2
\end{gathered}
$$

With these characterizations it is trivial to prove, for example, that

$$
\text { control }=\text { start } \Longrightarrow x \leq 5
$$

is inductive (and hence an invariant): it holds initially, and it is preserved by all discrete transitions and all time $(d)$ steps.

The above quantifier elimination can be carried in general under some reasonable assumptions. If $\varphi(\vec{v})$ is a state predicate then we write $\operatorname{post}(\varphi)(\vec{v})$ for the state predicate that holds for states that have a time predecessor satisfying $\varphi$ :

$$
\operatorname{post}(\varphi)(\vec{v}) \triangleq \exists \vec{w} \exists e>0:(\vec{v}=\vec{w} \oplus e) \wedge \varphi(\vec{w})
$$

If $\varphi$ only involves lower bounds on clocks, then $\operatorname{post}(\varphi)$ can typically be obtained from $\varphi$ by making these lower bounds strict, so quantifier elimination from $\operatorname{post}(\varphi)$ is easy. Hence, if preconditions and urgency predicates only involve lower bounds on clocks (which appears to be a good specification style anyway), then their conjunction is stable one may use the following lemma to eliminate the quantification over $e$ from the time progress predicate (7).

Lemma 2 Let $\varphi$ be a state predicate that is stable under time progress. Then

$$
(\forall 0 \leq e<d: \neg \varphi(\vec{v} \oplus e)) \Leftrightarrow \neg \operatorname{post}(\varphi)(\vec{v} \oplus d)
$$

Proof: Equivalence (9) can be rewritten into

$$
(\exists 0 \leq e<d: \varphi(\vec{v} \oplus e)) \Leftrightarrow \operatorname{post}(\varphi)(\vec{v} \oplus d)
$$

We prove both implications:

$\Rightarrow$ Assume $\varphi(\vec{v} \oplus e)$, for certain $e \in[0, d)$. Then $\operatorname{post}(\varphi)(\vec{v} \oplus d)$ holds since there is a state, namely $\vec{v} \oplus e$, that is a time predecessor of $\vec{v} \oplus d$ and in which $\varphi$ holds.

$\Leftarrow$ Assume $\operatorname{post}(\varphi)(\vec{v} \oplus d)$. Then by (8) there exists an $e^{\prime}>0$ and a state $\vec{w}$ such that $\varphi(\vec{w})$ holds and $\vec{v} \oplus d=\vec{w} \oplus e^{\prime}$. Depending on the relationship between $d$ and $e^{\prime}$ we have two cases:

1. Case $d<e^{\prime}$. Then $\vec{v}=\vec{w} \oplus\left(e^{\prime}-d\right)$. Since $\varphi(\vec{w})$ holds and $\varphi$ is stable under time progress, also $\varphi(\vec{v})$ holds. So we may choose $e=0$ to obtain $\varphi(\vec{v} \oplus e)$.

2. Case $e^{\prime} \leq d$. Then $\vec{v} \oplus\left(d-e^{\prime}\right)=\vec{w}$. Choose $e=d-e^{\prime}$. Then $d \in[0, d)$ and $\varphi(\vec{v} \oplus e)$, as required. 


\section{Concluding Remarks}

In this article, we introduced a notion of urgency predicates and compared it with three other constructs for specifying progress properties that have been proposed in the literature: invariants, stopping conditons and deadlines. We showed that under some rather realistic assumptions (use of clock variables, no strict upper bounds on progress, absence of time deadlocks,...) the four notions are equally expressive. Nevertheless, a clear advantage of deadlines and urgency predicates in practice is that one gets absence of time deadlocks (time reactivity) for free. A potential advantage of invariants is that they allow one to bound the time at which a (locally controlled) action occurs by a right-open interval. However, we are not aware of practical applications in which this feature is really needed. We argued that if one uses a precondition/effect style specification language, urgency predicates lead to shorter and more natural specifications than any of the other constructs, in particular invariant. In the graphical syntax used by e.g. UPPAAL, the use of urgency/deadline predicates would not lead to shorter specifications than the use of invariants. Typically, in first case one will decorate an edge of the graph (i.e., a transition) with a label $x \geq 4$, and in the second case a label $x \leq 4$ will be attached to a vertex of the graph (i.e., a location). But whereas the use of invariants may easily lead to time deadlocks, urgency/deadline predicates only stop time if there is a good reason for it, that is a specific transition that must be taken, and in this manner time deadlocks are avoided.

Folklore has it that urgency/deadline predicates are more difficult to implement in model checkers than invariants because they easily lead to non-convex zones. Non-convex zones indeed arise in the implementation of timed automata with deadlines in the IF toolset [6]. In particular, time transitions may lead from one convex zone to several convex zones (not only one, as in standard timed automata with invariants). When such a situation

arises in IF, the non-convex zone is automatically split in several, possibly overlapping, convex zones. The main reason why non-convex zones do not arise in standard timed automata with invariants (such as those implemented in UPPAAL) is that rather strong restrictions are imposed on the syntax of invariants. Only conjunctions of upper bounds on clocks - where the bounds are given by integer expressions - are allowed. If similar restrictions would be imposed in a syntax for urgency predicates, then no non-convex zones would arise in that setting either! More specifically, one would have to require that each urgency predicate is the disjunction of lower bounds on clocks, where the (non-strict) bounds are given by integer expressions. In addition, the urgency predicate of an input action $a$ ? should always be false. We think it would be a clear improvement to the current version of UPPAAL (3.4.7) to add such a restricted notion of urgency predicates to the syntax, replacing the notion of an urgent channel. Adding general urgency 
predicates to UPPAAL would of course also be a possibility, but this would require splitting of zones as in the IF toolset.

In the setting that we studied, urgency predicates appear to be a very nice way to specify progress properties, with clear advantages over some other constructs that have been advocated in the literature. Some remaining questions for future research are:

1. Exploration of proof rules to reason with urgency predicates in simulations and liveness proofs.

2. Establish versions of the compositionality results of $[5,19,3]$ in the setting of this paper.

3. Extension of our specification language and expressiveness results to a hybrid setting in which besides clocks also other continuously evolving variables are allowed.

\section{Acknowledgements}

Thanks to Nancy Lynch and Dilsun Kaynar for detailled comments on an earlier version of this note, and to Marius Bozga for answering some of our questions about IF.

\section{References}

[1] R. Alur and D.L. Dill. A theory of timed automata. Theoretical Computer Science, 126:183-235, 1994.

[2] R. Alur and T.A. Henzinger. Real-time system $=$ discrete system + clock variables. In T. Rus and C. Rattray, editors, Theories and Experiences for Real-Time System Development - Papers presented at First AMAST Workshop on Real-Time System Development, Iowa City, Iowa, November 1993, pages 1-29. World Scientific, 1994.

[3] S. Bornot, G. Gößler, and J. Sifakis. On the construction of live timed systems. In Susanne Graf and Michael I. Schwartzbach, editors, 6th International Conference on Tools and Algorithms for the Construction and Analysis of Systems, volume 1785 of Lecture Notes in Computer Science, pages 172-202. Springer-Verlag, April 2000.

[4] S. Bornot, J. Sifakis, and S. Tripakis. Modeling urgency in timed systems. In Willem P. de Roever, Hans Langmaack, and Amir Pnueli, editors, COMPOS, volume 1536 of Lecture Notes in Computer Science, pages 103-129. Springer, 1998. 
[5] Howard Bowman. Modelling timeouts without timelocks. In ARTS'99, 5th International AMAST Workshop on Real-time and Probabilistic Systems, LNCS, page 20. Springer-Verlag, May 1999.

[6] M. Bozga, S. Graf, and L. Mounier. IF-2.0: A validation environment for component-based real-time systems. In K.G. Larsen Ed Brinksma, editor, Proceedings of CAV'02, volume 2404 of $L N C S$, pages 343-348, Copenhagen, Denmark, July 2002. Springer.

[7] Pedro R. D'Argenio. A compositional translation of stochastic automata into timed automata. Technical report, University of Twente, 2000. CTIT-00-08.

[8] S.J. Garland, N.A. Lynch, J. Tauber, and M. Vaziri. IOA user guide and reference manual, 2003. Available through URL http://theory.lcs.mit.edu/tds/ioa.html.

[9] T.A. Henzinger, X. Nicollin, J. Sifakis, and S. Yovine. Symbolic model checking for real-time systems. Information and Computation, 111:193244, 1994.

[10] D.K. Kaynar, N.A. Lynch, and S. Mitra. Specifying and proving timing properties with TIOA tools. In 25th IEEE International Real-Time Systems Symposium, Work in Progress Session (RTSS 2004 WIP), December 5-8, 2004, Lisbon, Portugal, 2004.

[11] D.K. Kaynar, N.A. Lynch, R. Segala, and F.W. Vaandrager. A framework for modelling timed systems with restricted hybrid automata. In Proceedings of the 24th International IEEE Real-Time Systems Symposium (RTSS03), December 3-5, 2003, Cancun, Mexico, pages 166-178. IEEE Computer Society Press, 2003. Full version available as [12].

[12] D.K. Kaynar, N.A. Lynch, R. Segala, and F.W. Vaandrager. The theory of timed I/O automata. Technical Report MIT-LCS-TR-917, MIT Laboratory for Computer Science, Cambridge, MA, 2003.

[13] K. G. Larsen, P. Pettersson, and W. Yi. UppaAl in a Nutshell. Int. Journal on Software Tools for Technology Transfer, 1(1-2):134-152, October 1997.

[14] N.A. Lynch, R. Segala, and F.W. Vaandrager. Hybrid I/O automata. Information and Computation, 185(1):105-157, 2003.

[15] N.A. Lynch and M.R. Tuttle. An introduction to input/output automata. CWI Quarterly, 2(3):219-246, September 1989.

[16] Z. Manna and A. Pnueli. Temporal Verification of Reactive Systems: Safety. Springer-Verlag, 1995. 
[17] M. Merritt, F. Modugno, and M. Tuttle. Time constrained automata. In J.C.M. Baeten and J.F. Groote, editors, Proceedings CONCUR 91, Amsterdam, volume 527 of Lecture Notes in Computer Science, pages 408-423. Springer-Verlag, 1991.

[18] C.M. Robson. TIOA and UppaAl. Master's thesis, Department of Electrical Engineering and Computer Science, Massachusetts Institute of Technology, Cambridge, May 2004. http://theory.lcs.mit.edu/tds/papers/Robson/thesis.ps.

[19] J. Sifakis. The compositional specification of timed systems - a tutorial. In N. Halbwachs and D. Peled, editors, Proceedings of the 11th International Conference on Computer Aided Verification, Trento, Italy, volume 1633 of Lecture Notes in Computer Science, pages 2-7. SpringerVerlag, July 1999.

[20] J. Sifakis and S. Yovine. Compositional specification of timed systems (extended abstract). In Claude Puech and Rüdiger Reischuk, editors, STACS, volume 1046 of Lecture Notes in Computer Science, pages 347359. Springer, 1996.

[21] M.B. van der Zwaag and J. Hooman. A semantics of communicating reactive objects with timing. In Proceedings Workshop on Specification and Validation of UML models for Real-Time Embedded Systems (SVERTS 2003), pages 59-67. Verimag, 2003. http://www-omega.imag.fr/doc/d1000209_1/Semantics.pdf. 